\title{
Aprendizaje basado en proyectos en educación infantil: cambio pedagógico y social
}

\author{
Antonia Cascales Martínez; Mª Encarnación Carrillo García *
}

Resumen. El artículo presenta los resultados de una investigación cuyo propósito fue conocer la integración del Aprendizaje Basado en Proyectos en el segundo ciclo de Educación Infantil. A partir de un diseño de investigación ex post facto descriptivo, siendo la muestra participante 214 docentes de Educación Infantil de la Región de Murcia (España) que trabajan con ABP actualmente en sus aulas. Se utilizó un cuestionario para recoger datos confeccionado ad hoc, validado mediante el procedimiento de validación por juicios de expertos y la fiabilidad fue analizada mediante el alfa de Cronbach. Los resultados evidencian que el ABP es un instrumento muy efectivo para favorecer la justicia social, en la medida que su implementación respeta los ritmos de enseñanza y aprendizaje tanto de docentes como de alumnos. Asimismo, se destaca la dificultad de acoplarlo en el sistema dada la rigidez de las administraciones educativas.

Palabras clave: Aprendizaje Basado en Proyectos; educación infantil; evaluación; docentes; pedagogía social.

\section{APRENDIZAGEM FUNDADA EM PROJETOS DE EDUCAÇÃO INFANTIL: MUDANÇA PEDAGÓGICA E SOCIAL}

Resumo. O artigo apresenta os resultados de uma pesquisa cuja finalidade era conhecer a integração da Aprendizagem Baseada em Projetos no segundo ciclo da Educação Infantil. Este trabalho se baseia em um projeto de pesquisa descritiva ex post facto, onde a amostra participante foi de 214 professores de Educação Infantil da região de Múrcia (Espanha) que trabalham atualmente com ABP em suas salas de aula. Foi utilizado um questionário ad hoc para a coleta de dados, que foram validados pelo procedimento de validação mediante pareceres de especialistas, e a confiabilidade foi analisada por meio do método alfa de Cronbach. Os resultados proporcionam evidencias de que o ABP é um instrumento muito eficaz para promover a justiça social, na medida em que sua implementação respeita os ritmos de ensino e aprendizagem de professores e de alunos. Além disso, destaca-se a dificuldade de incluí-lo no sistema devido à rigidez das administrações educacionais.

Palavras-chave: Aprendizagem Baseada em Projetos; Educação Infantil; avaliação; professores; pedagogia social.

\footnotetext{
* Facultad de Educación, Universidad de Murcia (UM), España.
} 


\begin{abstract}
PROJECT-BASED METHOD IN CHILHOOD EDUCATION: SOCIAL PEDAGOGICAL CHANGE

Abstract. This paper presents the results of an investigation focus on knowing the Project-Based Method in Childhood Education. From an ex post facto descriptive investigation design, having a sample of 214 Childhood Education teachers of Región de Murcia (Spain) that currently works with this methodology. We used an ad hoc questionnaire, validated by experts, in order to collect the data. The reliability of the questionnaire was analyzed by the alpha of Cronbach. The results evidence that the Project-Based Method is an efective instrument that favours social justice, because its implementation respects the teaching and learning rhythmsof both, teachers and students. Likewise, it highlights the difficulty of embracing it because the rigid educational administration.
\end{abstract}

Keywords: Project-Based Method; childhood education; assessment; teachers; social pedagogy.

\title{
1. INTRODUCCIÓN
}

Exponemos en nuestra investigación, los resultados obtenidos acerca del Aprendizaje Basado en Proyectos (ABP) en la etapa de Educación Infantil, un enfoque metodológico que implica un cambio de paradigma pedagógico escolar. En el sentido en el que afecta tanto al proceso de enseñanza como de aprendizaje. Ello conlleva una nueva configuración del centro en todas sus dimensiones, requiere recursos y, además, supone una innovación del proceso de enseñanza-aprendizaje. En nuestro estudio, que se ha realizado durante el curso 2015-2016, han participado docentes de Educación Infantil de centros de la Región de Murcia (España), y se ha estudiado la incidencia de la metodología ABP en el aula.

\section{FUNDAMENTACIÓN TEÓRICA}

Gallardo, Ríos, Fernández, y Ramos (2016) explican que no hay una única manera de mirar la escuela de la infancia, desde su perspectiva la Educación Infantil se concibe como centro de vida, de juego, de comunicación y de conocimiento, en definitiva, un espacio diseñado y organizado para hacer posible una existencia gozosa, con múltiples y variadas experiencias de relación y de realización personal. Desde esta perspectiva, el Aprendizaje Basado en Proyectos (ABP) implica un cambio de paradigma pedagógico frente a los modelos tradicionales de enseñanza-aprendizaje. Dichos modelos, herencia de otros tiempos, en los que no se tenían en cuenta las características psicoevolutivas del alumnado, los diferentes ritmos de aprendizaje 
individuales ni el entorno social de procedencia; convertían las clases en sesiones expositivas donde los aprendices eran expuestos a los contenidos de forma pasiva, a modo de recipientes vacíos, donde el saber era depositado.

Hoy sabemos, avalados por múltiples estudios, el carácter relevante de los aspectos sociales en los procesos educativos, Vygotsky (1982) destacó el origen social de los procesos psicológicos incidiendo en que se forman en "la fase social" que las personas establecen con los objetos y en contacto con el resto de individuos; tal y como describe en su Ley genética del desarrollo cultural" en la que explica que en el desarrollo cultural del niño, toda función aparece dos veces, primero en el ámbito social y, más tarde, en el ámbito individual, esto es, primero entre personas (interpsicológica) y después en el interior del propio niño (intrapsicológica). También sabemos que respetar los ritmos de aprendizaje individuales, favorece el concepto de justicia social (Bolivar, 2012; Latapí, 2012), lo que convierte el aula en un espacio educativo democrático de integración de las personas, sean cuales sean sus características personales y sus necesidades educativas. En esta línea, Fernández y Gallardo (2016), entienden el aula como contexto social de aprendizaje, y explican que la escuela es un magnífico espacio para crecer como ser social, para desarrollar habilidades comunicativas, para aprender a expresarse siendo entendido por los demás, para compartir y para aprender a vivir y convivir, pues reflexionan que es imposible imaginar un mundo de aislamiento, sin relaciones y sin grupo.

Belavi y Murillo (2016) revisan en su estudio diversos conceptos relacionados con la educación, la democracia y la justicia social; y abogan por una educación de todos y para todos, que fomente el desarrollo integral del alumnado, a la vez que se atiende de forma individualizada a cada uno de ellos, con el fin de respetar sus ritmos de aprendizaje. Y para ello, explican que es necesaria una educación crítica, que reflexione acerca del porqué de las cosas, con unos docentes -intelectuales críticos- que fomenten en los estudiantes, cambios encaminados a la justicia social, mediante una educación democrática. En este sentido las metodologías utilizadas en el aula, que respeten a cada una de las personas que se encuentren en ella, además de favorecer un tipo de aprendizaje que fomente la crítica, la colaboración, y la búsqueda de respuestas activa mientras se realiza un proyecto común, ayudarán en gran medida a este propósito.

Trabajar estos conceptos en nuestras aulas implica empezar desde las bases de la escolarización y terminar en la enseñanza superior; buscando remover los cimientos del aula tradicional donde la homogeneidad se establece por rutina, independientemente del potencial individual de los individuos, y donde el adoctrinamiento puede llegar a primar sobre el aprendizaje y la creatividad. En contraposición, los entornos de aprendizaje que fomenten la 
colaboración entre el alumnado, y con metodologías activas, donde se aprende haciendo y en la acción, favorecerá el nuevo planteamiento educativo que exponemos.

A su vez, este nuevo paradigma implica trabajar en torno a un nuevo concepto de centro educativo, en relación a todas sus dimensiones y agentes, en la medida que es necesario diseñar y programar de forma diferente los objetivos, contenidos y criterios de evaluación; y configurar los espacios de enseñanza que favorezcan el ABP, así como la utilización de una serie de recursos educativos en consonancia con esta metodología tenida aun hoy como innovadora, y propiciar el oportuno cambio de los procesos educativos y ofrecer una alternativa a las "clases tradicionales".

Los procesos de enseñanza y aprendizaje en la etapa de Educación Infantil, el primer escalafón educativo, donde el alumnado participa de forma activa mientras aprende, se pueden ver beneficiados en la medida que utilizamos herramientas educativas adecuadas. En esta investigación, abordaremos el ABP desde esta perspectiva, tomándolo como una herramienta educativa que busca promover el aprendizaje significativo del alumnado de forma activa y colaborativa, y que facilite que los alumnos se involucren en su propio proceso de aprendizaje como un agente más del proceso educativo.

En 1918 William Heard Kilpatrick, en su artículo The Project Method: The Use of the Purposeful Act in the Educative Process, estableció las bases del ABP que hoy en día conocemos, las cuales favorecen el aprendizaje a la vez que se respetan las capacidades innatas de los niños, poniendo énfasis en el papel activo del alumnado en el proceso de enseñanza-aprendizaje.

Los estudios al respecto desde entonces han sido numerosos, el panorama educativo ha ido cambiando, pero aún queda mucho por hacer, debido a que los cambios son lentos y poco profundos. Vergara (2015) enumera en su obra las bases del ABP; y explica además, con ejemplos prácticos basados en su propia experiencia a través de los años, el potencial del ABP para crear un nuevo marco educativo, definiéndolo (Vergara, 2015: 146) como un proceso de aprendizaje basado en la investigación, los principios de negociación igualitaria, la responsabilidad individual, la actitud de cooperación y la toma de decisiones consensuada.

Helm y Katz (2016), en su obra acerca del ABP en edades tempranas, revisan el cómo empezar, desarrollar y concluir un proyecto de investigación por parte de los alumnos en la teoría y en la práctica; e inciden que este enfoque metodológico favorece la curiosidad hacia el aprendizaje de los nuevos contenidos y se adapta a los ritmos de aprendizaje del alumnado, ya que 
investigar un tema en profundidad ayuda a alcanzarlos objetivos educativos programados, a la vez que favorece la atención a la diversidad y la inclusión del alumnado.

En esta línea, Balongo y Mérida (2016) analizan en su investigación aspectos relacionados con la educación inclusiva en un aula de Educación Infantil de un colegio público de Córdoba (España), donde trabajan por proyectos; y en concreto estudian la ayuda entre iguales que se da, el incremento de la motivación, la fuerte implicación emocional que se genera, la atención personalizada que se establece, y la relación entre discentes y docente; concluyendo que esta metodología contribuye a la integración educativa de los estudiantes, lo cual significa un paso previo e imprescindible para la construcción de un clima escolar positivo.

El papel de las familias en los procesos de enseñanza juega un papel primordial, de manera que aquellas metodologías que promuevan su inclusión, se pueden ver también favorecidas como apuntan Ruiz y Mérida (2016) y Balongo y Mérida (2016). Trabajar con ABP en Educación Infantil, conlleva una reestructuración de las relaciones que se establecen entre todos los actores de la comunidad educativa (Cascales-Martínez, CarrilloGarcía y Redondo-Rocamora, 2017). En este sentido se puede decir que, según apuntan Balongo y Mérida (2016), la metodología ABP utiliza como parte esencial del proceso de enseñanza-aprendizaje los conocimientos y experiencia profesional de las familias, pues las familias asumen un papel de tutorización y acompañamiento en los procesos educativos de sus hijos e hijas a la vez que se dan en la escuela, consiguiéndose un compromiso elevado de las familias, las cuales lo valoran positivamente en la medida que entienden que el ABP es una metodología que "escucha" al alumnado, en relación a la atención individualizada que se le da.

Existen investigaciones que analizan la puesta en práctica del ABP, en relación a una materia determinada como vemos en Ortí e Iniesta-Sepúlveda (2016), en concreto se analiza el ABP como propuesta metodológica para la mejora de la didáctica de las matemáticas en la etapa de Educación Infantil, utilizando también como recurso educativo la música, haciendo de ella, de modo global e interdisciplinar, una herramienta innovadora para que el alumnado adquiera contenidos relacionados con el currículo de Educación Infantil.

La puesta en práctica del ABP en condiciones óptimas también necesita una organización de los espacios, los materiales y los recursos determinada, así como por la inclusión de las Tecnologías del Aprendizaje y la Comunicación, de manera que esta nueva metodología implique un cambio paradigmático efectivo. Chávez, Cantú y Rodríguez (2016) explican en su investigación sobre el ABP en relación a las competencias digitales que se 
promueven en Educación Infantil, que dicho estudio responde a la necesidad de determinar un entorno educativo donde se integren el ABP y las Tecnologías de la Información y la Comunicación (TIC), concluyendo que se pueden obtener resultados satisfactorios si se involucra activamente a los alumnos con el ABP y con el apoyo de las TIC.

Mosconi, Gamino y González (2016) en su artículo sobre el aula extendida, nos hablan de la integración de las tecnologías de la información en relación con el $A B P$, en concreto, sobre las redes sociales y su papel en los procesos educativos relacionados con el $A B P$, ya que favorecen la integración global de los contenidos trabajados en los procesos de investigación de los alumnos que realizan un proyecto, visibilizándolos ante el resto de la comunidad educativa.

Moral, Villalustre y Neira (2013) explican en su estudio que las Tecnologías de la Información y la Comunicación son agentes motivadores que permiten al alumnado una inmersión en el ámbito tecnológico que les ayuda a romper con la brecha existente entre medio rural y urbano, potenciando sus competencias digitales, capacitándolo para la búsqueda y tratamiento de la información, y el manejo de herramientas informáticas para la comunicación y el desarrollo de los proyectos colaborativos. Por ello, de sus palabras destacamos que, los entornos rurales aislados ven enriquecidos los procesos educativos en los que están involucrados los proyectos del tipo colaborativo mediante la metodología ABP y las tecnologías, en la medida que las tecnologías favorecen la investigación, el acceso a la información y la colaboración entre otros compañeros y centros educativos.

EI ABP en Educación Infantil es un recurso metodológico que permite el desarrollo de las capacidades de esa etapa psicoevolutiva, y además permite que el alumnado pueda desenvolverse con éxito en situaciones cotidianas. Esta premisa orienta los problemas planteados en esta investigación, y nos lleva a preguntarnos: ¿cómo diseñan el ABP los docentes que trabajan en Educación Infantil?, ¿cuáles son los criterios que utilizan para la selección de objetivos y contenidos?, ¿qué estrategias ponen en marcha a la hora de trabajar con ABP?, ¿se contempla la evaluación en este tipo de metodología?, ¿las TIC se asocian al ABP?, ¿el ABP tiene carácter innovador?, ¿tiene alguna implicación el tipo de centro educativo al que pertenecen?, ¿el grado de formación en TIC?

Por tanto, este estudio tiene como objetivo conseguir una fotografía sobre cómo el ABP está integrado en el proceso de enseñanza en la etapa de Educación Infantil en la Región de Murcia. Dado que el cambio metodológico, en relación al $A B P$, es un proceso lento y complejo e implica muchos factores. En concreto, este estudio pretende, por un lado, analizar el planteamiento 
teórico del ABP en torno a la práctica profesional en Educación Infantil, y por otro, dar respuesta a las cuestiones relacionadas con su implementación en el aula.

\section{MÉTODO}

El estudio realizado se enmarca dentro en una metodología cuantitativa adoptando un planteamiento descriptivo-comparativo con diseño no experimental de grupo único tipo encuesta (Gay y Airasian, 2000), cuya finalidad exploratoria trata de conocer el desarrollo del ABP en el segundo ciclo de la Etapa de Educación Infantil en el contexto de la implementación de este modelo pedagógico.

Esta investigación recoge una muestra pequeña de docentes y por tanto no está exento de limitaciones, si bien los hallazgos pueden tener implicaciones para el desarrollo del pensamiento teórico sobre el profesional de Educación Infantil. El objeto del estudio no es la generalización de los resultados, sino el análisis de una muestra de docentes que trabajan con ABP para analizar la realidad y proponer formación ajustada a las necesidades del docente de Educación Infantil en activo.

\subsection{Participantes}

El procedimiento de selección de la muestra ha sido no probabilístico intencional, en la medida que se ha aplicado la encuesta solamente a docentes de Educación Infantil que trabajan en sus aulas con ABP.

El cuestionario se envió de forma personalizada a un total de 350 docentes que impartían docencia en el segundo ciclo de Educación Infantil en la Región de Murcia de los cuales han respondido 214.

En lo que se refiere a la formación académica, los docentes son mayoritariamente especialistas en Educación Infantil (88,3\%). De los participantes, el $68,8 \%$ son maestras y $31,2 \%$ son maestros. La edad de los docentes, el $79,6 \%$ se encuentra entre los 26 y 45 años. En cuanto a la titularidad del centro en el que trabajan, el 79,4\% de los docentes participantes pertenecen a centros públicos, y el 20,6 \% en centros privadosconcertados. En cuanto al nivel en el que imparten docencia actualmente, el 38,8 \% lo hacen en el aula de 3 años, el 13,1 \% en el aula de 4 años, $22,4 \%$ en 5 años y el 25,7\% actúa como docente de apoyo. En el caso de la experiencia laboral de los docentes, el 46,4 \% manifiesta haber trabajado 
menos de tres años. También el 59,6\% de los participantes considera que su formación en TAC es a nivel de usuario, frente al 14,6\% que lo considera básico. Finalmente, el 44,7 \% lleva trabajando con ABP más de tres años, frente a un $41,3 \%$ que solamente lleva un año.

\subsection{Instrumentos}

Los datos del estudio han sido recogidos a través de una encuesta diseñada ad hoc. La estructura del instrumento se compone de dos partes: en la primera parte se solicitó información sobre variables demográficas: titulación, género, edad, experiencia laboral, grado de formación en TAC, titularidad del centro, curso en el que imparten docencia actualmente y años de trabajo con ABP. La segunda parte se compone de 35 ítems agrupados en las siguientes dimensiones: diseño de los proyectos de ABP, en qué medida se ajustan los objetivos y contenidos a este tipo de metodología, aspectos relacionados con la metodología, valoración de la evaluación como parte del ABP y, finalmente, se refiere a la formación del profesorado en el uso adecuado del ABP. La forma de respuesta es una escala tipo Likert, de valoración de cuatro puntos (de 1="1-Totalmente en desacuerdo" a 4="Totalmente de acuerdo)

La propia naturaleza del instrumento aconsejaba su validación de contenido con una estrategia de fiabilidad entre jueces de carácter cualitativo, para lo cual se contó con cinco expertos: una doctora en pedagogía y experta en ABP en Educación Infantil, una profesora titular de universidad y experta en tecnologías educativas, una docente de Educación Infantil, una docente en Educación Primaria y una asesora de formación en Educación Infantil y Primaria. Se solicitó a los expertos que valorasen diferentes aspectos sobre los datos iniciales sociodemográficos, las dimensiones, los ítems, opciones de respuesta, y una valoración global (Wieserma, 2001). Tras dicha validación se realizaron mínimas aportaciones al cuestionario como fueron la incorporación de una respuesta a una de las preguntas cerradas y algunas modificaciones de redacción.

El análisis de la fiabilidad del instrumento, ha obtenido una alta consistencia interna, arrojando un coeficiente de Alfa de Cronbach de 0,946 siendo, según De Vellis (2003), excelente al obtener una puntuación mayor de 0,90 .

\subsection{Diseño y análisis de datos}

La creación de la base de datos y el análisis posterior cuantitativo de la información se ha realizado con el paquete estadístico IBM SPSS Statistic (vers. 19.0) para Windows y ha consistido en el cálculo de estadísticos 
descriptivos, Alfa de Cronbach. Para la comparación de medias se utilizó la prueba t para muestras independientes y ANOVA simple, y por último estadísticos de relación entre variables. El nivel de significatividad para el estudio se ha establecido el $p<0.05$.

\subsection{Resultados: análisis descriptivo de las distintas categorías}

Para el análisis de los resultados descriptivos se va a seguir la estructura de las dimensiones que conforman el cuestionario: diseño, objetivos y contenidos, metodología, evaluación, formación, tecnología e innovación.

\subsubsection{Diseño de proyecto de ABP}

En la Tabla 1 podemos observar las medidas de tendencia central y dispersión sobre el nivel de conocimiento que el profesorado indica tener con respecto al diseño de los proyecto de ABP.

\section{TABLA 1}

\section{Descriptivos de tendencia central y dispersión en el diseño de ABP}

\begin{tabular}{|l|c|c|c|c|}
\hline & Media & Mediana & Moda & D. Típ. \\
\hline $\begin{array}{l}\text { Conozco los fundamentos psicológicos, pedagógicos, } \\
\text { sociales y epistemológicos del ABP. }\end{array}$ & 2,22 & 2 & 2 & 0.92 \\
\hline $\begin{array}{l}\text { El ABP permite dar respuesta a la complejidad del } \\
\text { proceso de enseñanza-aprendizaje. }\end{array}$ & 3,29 & 3 & 3 & 0,73 \\
\hline Para trabajar ABP me apoyo en editoriales comerciales. & 1,71 & 1 & 1 & 0,95 \\
\hline Soy capaz de diseñarygestionar mis propios proyectos. & 3,12 & 3 & 3 & 0,78 \\
\hline $\begin{array}{l}\text { Trabajar con ABP me permite actualizar mis conoci- } \\
\text { mientos y competencias docentes. }\end{array}$ & 3,36 & 4 & 4 & 0,82 \\
\hline $\begin{array}{l}\text { Trabajar con ABP me permite integrar innovaciones } \\
\text { en el aula. }\end{array}$ & 3,55 & 4 & 4 & 0,76 \\
\hline $\begin{array}{l}\text { La programación con ABP me limita a la hora de } \\
\text { enseñar y programar mi actuación docente. }\end{array}$ & 3,48 & 4 & 4 & 0,80 \\
\hline $\begin{array}{l}\text { Tengo destrezas para averiguar las necesidades de } \\
\text { mis alumnos previos a plantear un proyecto de ABP. }\end{array}$ & 2,82 & 3 & 3 & 0,77 \\
\hline $\begin{array}{l}\text { Los proyectos de ABP suelen ser los mismos para } \\
\text { todos los alumnos del ciclo de Educación Infantil. }\end{array}$ & 2,09 & 2 & 2 & 0,85 \\
\hline
\end{tabular}

A la hora de diseñar el ABP, los docentes participantes indican, según se muestra en la Tabla 1 , que trabajar con esta metodología les permite integrar innovaciones en el aula $(M=3,55)$, a la vez que reconocen que la programación con $\mathrm{ABP}$ apenas les limita a la hora de enseñar y programar su actuación docente $(M=3,48)$, a la vez que les ayuda a actualizar sus conocimientos y competencias docentes $(M=3,36)$, y les posibilita dar respuesta a 
la complejidad del proceso de enseñanza-aprendizaje $(M=3,29)$. Asimismo, se puede apreciar que los docentes que han participado en este estudio no se apoyan para el diseño de ABP en las editoriales comerciales $(M=1.71)$ hecho que también se refleja con la puntuación obtenida en la moda (1).

\subsubsection{Objetivos y contenidos}

En cuanto a la selección de objetivos y contenidos dentro de la metodología de $\mathrm{ABP}$, los datos descriptivos de tendencia central y de dispersión se muestran en la Tabla 2.

\section{TABLA 2}

Descriptivos de tendencia central y dispersión en los objetivos y contenidos de ABP

\begin{tabular}{|l|c|c|c|c|}
\hline & Media & Mediana & Moda & D. Típ. \\
\hline $\begin{array}{l}\text { Diseño los objetivos del un proyecto de ABP con el } \\
\text { resto de compañeros de ciclo. }\end{array}$ & 2,85 & 3 & 3 & 0,97 \\
\hline $\begin{array}{l}\text { Diseño los objetivos del un proyecto de ABP con el } \\
\text { equipo docente de mi aula. }\end{array}$ & 2,78 & 3 & 3 & 1,01 \\
\hline Procuro dar contenidos integradosen un marcogeneral. & 3,13 & 3 & 3 & 0,66 \\
\hline $\begin{array}{l}\text { Los contenidos teóricos pierden peso en pro de los } \\
\text { procedimentales y actitudinales. }\end{array}$ & 2,62 & 3 & 2 & 0,99 \\
\hline
\end{tabular}

Los resultados que se muestran indican que los docentes que trabajan con ABP y han participado en este estudio procuran dar contenidos integrados en un marco general $(M=3.13)$. El resto de ítems han obtenido medias superiores al 2,50 y la moda en los ítems sobre objetivos y contenidos es de tres sobre cuatro, excepto en el ítem que evalúa el peso de contenidos teóricos en relación a los procedimentales y actitudinales que obtiene una moda de 2.

\subsubsection{Metodología}

Respecto a los aspectos metodológicos, según los datos recogidos en la Tabla 3, el profesorado de la muestra indica que trabajar con ABP posibilita reconocer el mérito a los alumnos cuando han realizado un buen trabajo $(M=3,59)$. Asimismo, los docentes reconocen que el $A B P$ ayuda a plantear actividades que sean más atractivas, vistosas y audiovisuales $(M=3,48)$. 


\section{TABLA 3}

\section{Descriptivos de tendencia central y dispersión en la metodología de ABP}

\begin{tabular}{|l|c|c|c|c|}
\hline & Media & Mediana & Moda & D. Típ. \\
\hline $\begin{array}{l}\text { En un proyecto de ABP solamente trabajas en función } \\
\text { de lo planificado. }\end{array}$ & 1,83 & 2 & 2 & 0,87 \\
\hline $\begin{array}{l}\text { Tengo dificultades para cambiar de estrategias } \\
\text { metodológicas. }\end{array}$ & 2,14 & 2 & 2 & 0,75 \\
\hline Favorezco el aporte de ideas sin limitación formal. & 2,79 & 3 & 3 & 0,86 \\
\hline $\begin{array}{l}\text { Las actividades planificadas trato de que no sean } \\
\text { repetitivas. }\end{array}$ & 2,82 & 3 & 3 & 0,87 \\
\hline $\begin{array}{l}\text { Las dudas que surgen se anteponen a la planifica- } \\
\text { ción curricular. }\end{array}$ & 2,77 & 3 & 3 & 0,93 \\
\hline Doy prioridad a los intereses de mis alumnos. & 3,31 & 3 & 4 & 0,78 \\
\hline $\begin{array}{l}\text { Procuro controlar a los alumnos evitando interven- } \\
\text { ciones espontaneas. }\end{array}$ & 3,29 & 3 & 4 & 0,77 \\
\hline $\begin{array}{l}\text { Suelo proponer a los alumnos que resuelvan activi- } \\
\text { dades de forma voluntaria. }\end{array}$ & 3,24 & 3 & 3 & 0,61 \\
\hline $\begin{array}{l}\text { Propongo actividades que necesitan buscar infor- } \\
\text { mación y sacar conclusiones. }\end{array}$ & 3,30 & 3 & 3 & 0,58 \\
\hline $\begin{array}{l}\text { Propongo actividades para que los alumnos trabajen } \\
\text { en equipo. }\end{array}$ & 3,33 & 3 & 3 & 0,69 \\
\hline $\begin{array}{l}\text { Suelo llevar expertos en la materia al aula para que } \\
\text { los alumnos aprendan mejor. }\end{array}$ & 2,98 & 3 & 3 & 0,84 \\
\hline $\begin{array}{l}\text { Reconozco el mérito de los alumnos cuando han } \\
\text { realizado un buen trabajo. }\end{array}$ & 3,59 & 4 & 4 & 0,75 \\
\hline $\begin{array}{l}\text { El ABP posibilita que las actividades que se de- } \\
\text { sarrollan en las clases puedan ser más atractivas, } \\
\text { vistosas y audiovisuales. }\end{array}$ & 3,48 & 4 & 4 & 0,72 \\
\hline $\begin{array}{l}\text { Combino metodologías que requieran el trabajo } \\
\text { colaborativo e individualizado. }\end{array}$ & 3,29 & 4 & 4 & 0,86 \\
\hline $\begin{array}{l}\text { El ABP me permite dar una respuesta educativa de } \\
\text { calidad a los alumnos de necesidades educativas } \\
\text { especiales. }\end{array}$ & 3,31 & 3 & 4 & 0,78 \\
\hline
\end{tabular}

En esa misma línea, los docentes indican que el ABP les permite, por un lado, dar una respuesta educativa de calidad a los alumnos de necesidades educativas especiales ( $M=3,31)$; y por otro lado, combinar metodologías que requieran el trabajo colaborativo e individualizado $(M=3,29)$.

En cuanto al diseño de actividades, los docentes indican que suelen proponer actividades para que los alumnos trabajen en equipo $(M=3,33)$, actividades que necesiten buscar información y sacar conclusiones $(M=3,30)$ y actividades de resolución voluntaria $(M=3,24)$.

Finalmente, el profesorado reconoce que en un proyecto de $A B P$ no solamente se trabaja en función de lo planificado $(M=1.83)$, hecho que concuerda con la misma filosofía de partida propuesta por Killpatrick (1918). 


\subsubsection{Evaluación}

Se preguntó a los docentes encuestados sobre la evaluación con $A B P$, dado que es un elemento fundamental dentro del proceso de enseñanza- aprendizaje.

\section{TABLA 4}

Descriptivos de tendencia central y dispersión en la evaluación de ABP

\begin{tabular}{|l|c|c|c|c|}
\hline $\begin{array}{l}\text { Las evaluaciones con ABP valoran los contenidos } \\
\text { abordados en el proyecto. }\end{array}$ & 3,09 & 3 & 3 & 0,81 \\
\hline $\begin{array}{l}\text { Las evaluaciones con ABP valoran los contenidos } \\
\text { exclusivamente curriculares. }\end{array}$ & 1,81 & 1 & 1 & 0,77 \\
\hline $\begin{array}{l}\text { EI ABP me permite utilizar diferentes técnicas para } \\
\text { evaluar a los alumnos. }\end{array}$ & 3,34 & 2 & 1 & 0,74 \\
\hline $\begin{array}{l}\text { EI ABP me permite utilizar diferentes instrumentos } \\
\text { para evaluar a los alumnos. }\end{array}$ & 3,37 & 3 & 4 & 0,79 \\
\hline $\begin{array}{l}\text { Los resultados de los alumnos son mejores cuando } \\
\text { se trabaja con ABP. }\end{array}$ & 2,96 & 4 & 4 & 0,79 \\
\hline
\end{tabular}

Los resultados obtenidos en estos ítems (véase Tabla 4) muestran que los docentes que han trabajado con ABP utilizan diferentes instrumentos $(M=3,37)$ y técnicas $(M=3,34)$ para evaluar a los alumnos y valoran las contenidos abordados en el proyecto $(M=3,03)$. Toda vez que reconocen que las evaluaciones con ABP no valoran las contenidos exclusivamente curriculares $(\mathrm{M}=1.81)$.

\subsubsection{Formación}

En otra dimensión abordamos la formación recibida por los docentes en torno al ABP, se les hizo varias preguntas al respecto, destacando los siguientes resultados en la Tabla 5:

\section{TABLA 5}

Descriptivos de tendencia central y dispersión en la formación de ABP

\begin{tabular}{|l|c|c|c|c|}
\hline & Media & Mediana & Moda & D. Típ. \\
\hline La formación en ABP es autónoma. & 2,49 & 2 & 2 & 0,86 \\
\hline Asisto a cursos de formación sobre ABP. & 2,55 & 3 & 3 & 1,00 \\
\hline $\begin{array}{l}\text { Participo en proyectos de innovación/investigación } \\
\text { relacionados con el ABP. }\end{array}$ & 2.89 & 3 & 4 & 1,08 \\
\hline
\end{tabular}

Los docentes participantes, respecto a la formación recibida en ABP, se puede observar en la Tabla 5 que obtienen la formación participando en proyecto de innovación/ investigación relacionados con el $A B P(M=2.89)$. 
Las medias bajan cuando se les pregunta en qué medida asisten a cursos de formación sobre esta temática $(M=2.55)$ y la media es ligeramente inferior al hablar de formación en ABP como autónoma $(M=2.49)$.

\subsubsection{Tecnología}

Dentro del ABP la utilización de las tecnologías adquiere cada vez más importancia en el proceso enseñanza-aprendizaje, así preguntamos a los docentes sobre ello. Los resultados obtenidos por los docentes quedan recogidos en la Tabla 6:

\section{TABLA 6}

Descriptivos de tendencia central y dispersión en la utilización de las TIC en ABP

\begin{tabular}{|l|c|c|c|c|}
\hline & Media & Mediana & Moda & D. Típ. \\
\hline $\begin{array}{l}\text { Conozco las posibilidades de los recursos tecno- } \\
\text { lógicos para diseñar mis propias actividades y } \\
\text { presentaciones en mis proyectos de ABP. }\end{array}$ & 2.67 & 3 & 3 & 0.73 \\
\hline $\begin{array}{l}\text { Hago uso de los recursos tecnológicos para mejorar } \\
\text { mis proyectos de ABP. }\end{array}$ & 3.02 & 3 & 4 & 0.90 \\
\hline $\begin{array}{l}\text { Diseño actividades de aprendizaje utilizando } \\
\text { diferentes herramientas tecnológicas (ordenador, } \\
\text { PDI, tabletas ...). }\end{array}$ & 2.90 & 3 & 4 & 1.05 \\
\hline $\begin{array}{l}\text { Diseño contenidos educativos digitales realizados } \\
\text { en mis proyectos de ABP. }\end{array}$ & 2.63 & 3 & 2 & 0.99 \\
\hline $\begin{array}{l}\text { Recurro a los contenidos digitales que encuentro } \\
\text { en la red para mis proyectos de ABP. }\end{array}$ & 3.18 & 3 & 4 & 0.84 \\
\hline $\begin{array}{l}\text { Comparto los contenidos y actividades TIC con el } \\
\text { resto de la comunidad educativa. }\end{array}$ & 2.56 & 3 & 3 & 1.06 \\
\hline $\begin{array}{l}\text { Intento que las actividades planteadas a mi alum- } \\
\text { nado con recursos tecnológicos que despierten } \\
\text { su interés y les permita aprender de una manera } \\
\text { significativa. }\end{array}$ & 3.26 & 3 & 4 & 0.82 \\
\hline $\begin{array}{l}\text { Suelo descargar juegos y aplicaciones desde } \\
\text { internet, sin hacer ningún tipo de adaptaciones. }\end{array}$ & 2.46 & 2 & 2 & 0.84 \\
\hline $\begin{array}{l}\text { Aplico las normas de propiedad intelectual existen- } \\
\text { tes en cuanto al uso de los recursos y aplicaciones } \\
\text { tecnológicas. }\end{array}$ & 2.83 & 3 & 3 & 0.98 \\
\hline $\begin{array}{l}\text { Procuro utilizar recursos y/o aplicaciones que } \\
\text { permitan un trabajo colaborativo. }\end{array}$ & 3.11 & 3 & 4 & 0.90 \\
\hline
\end{tabular}

El análisis detallado de las respuestas indica que los participantes plantean actividades a su alumnado con recursos tecnológicos que despiertan su interés y les permiten aprender de una manera significativa $(M=3.18)$. También, recurren a contenidos digitales que encuentran en internet para trabajar con sus proyectos de ABP ( $M=3.18)$, a la vez que utilizan recursos y/o aplicaciones que permiten un trabajo colaborativo entre su alumnado 
$(\mathrm{M}=3.18)$ y hacen uso de los recursos tecnológicos para mejorar sus proyectos de $A B P(M=3.02)$. La media es más baja cuando se les pregunta en qué medida hacen adaptaciones de las aplicaciones que encuentran en internet y van a trabajar con sus alumnos $(M=2.46)$.

\subsubsection{Innovación}

Finalmente, dado que es una metodología actualmente muy relacionada con la innovación educativa, se les planteó varias cuestiones al respecto. Los resultados obtenidos se muestran en la Tabla 7:

\section{TABLA 7}

Descriptivos de tendencia central y dispersión sobre la innovación en ABP

\begin{tabular}{|l|c|c|c|c|}
\hline & Media & Mediana & Moda & D. Típ. \\
\hline $\begin{array}{l}\text { El ABP me ayuda a investigar en el aula sobre } \\
\text { diferentes temas con mis alumnos. }\end{array}$ & 2.61 & 3 & 3 & 0.69 \\
\hline $\begin{array}{l}\text { Utilizo el ABP para desarrollar el pensamiento } \\
\text { crítico de mis alumnos. }\end{array}$ & 3.13 & 3 & 4 & 0.98 \\
\hline $\begin{array}{l}\text { Tengo en cuenta cuándo es pertinente usar el ABP } \\
\text { y cuándo es mejor utilizar otro tipo de metodología. }\end{array}$ & 3.01 & 3 & 4 & 1.11 \\
\hline $\begin{array}{l}\text { Hago uso del ABP para diseñar centros de interés } \\
\text { innovadores. }\end{array}$ & 2.79 & 3 & 3 & 0.91 \\
\hline $\begin{array}{l}\text { Procuro acoplar nuevas metodologías al ABP dentro } \\
\text { del proceso de enseñanza-aprendizaje. }\end{array}$ & 3.22 & 3 & 4 & 0.88 \\
\hline $\begin{array}{l}\text { Adapto las nuevas herramientas o dispositivos tec- } \\
\text { nológicos que aparecen en el mercado a la realidad } \\
\text { de mi aula. }\end{array}$ & 2.55 & 3 & 3 & 1.08 \\
\hline $\begin{array}{l}\text { Utilizo el ABP con otros recursos TIC para mejorar } \\
\text { mi práctica docente. }\end{array}$ & 3.15 & 3 & 3 & 0.86 \\
\hline $\begin{array}{l}\text { Estudio nuevas metodologías que conlleven nuevos } \\
\text { planteamientos de ABP. }\end{array}$ & 2.55 & 2 & 2 & 0.80 \\
\hline $\begin{array}{l}\text { Participo en proyectos de innovación/ investigación } \\
\text { relacionados con el ABP. }\end{array}$ & 2.89 & 3 & 4 & 0.98 \\
\hline
\end{tabular}

Los docentes participantes, en general, asocian el ABP con la innovación educativa, y en muchos casos procuran dar un paso más acoplando nuevas metodologías al ABP dentro del proceso de enseñanza-aprendizaje $(M=3.22)$, a la vez que incorporan recursos TIC para mejorar su práctica docente $(\mathrm{M}=3.15)$ y utilizan esta metodología para desarrollar el pensamiento crítico de sus alumnos $(M=3.13)$. Sin embargo, las medias bajan cuando se les pregunta en qué medida el APB ayuda a investigar en el aula sobre diferentes temas ( $M=2.61$ ), si estudian nuevas metodologías que conlleven nuevos planteamientos de ABP y si adaptan las nuevas herramientas o dispositivos tecnológicos que aparecen en el mercado a la realidad de su aula $(M=2.55)$. 


\subsubsection{Comparación de los aspectos evaluados}

Finalmente, contrastamos las valoraciones de los docentes participantes con las dimensiones evaluadas, obteniendo los siguientes resultados (véase Tabla 8):

TABLA 8

Descriptivos de tendencia central y dispersión de los dimensiones valoradas

\begin{tabular}{|l|c|c|c|c|}
\hline & Media & Mediana & Moda & D. Típ. \\
\hline Diseño & 2,84 & 2 & 3 & 0,29 \\
\hline Objetivos y contenidos & 2,84 & 2 & 3 & 0,49 \\
\hline Metodología & 3,03 & 3 & 3 & 0,42 \\
\hline Evaluación & 2,91 & 3 & 2 & 0,42 \\
\hline Formación & 2,64 & 2 & 3 & 0,68 \\
\hline Tecnología & 2,86 & 2 & 2 & 0,62 \\
\hline Innovación & 2,87 & 2 & 2 & 0,64 \\
\hline
\end{tabular}

La dimensión mejor valorada por los docentes participantes en este estudio es la metodología ( $M=3.03$ ) destacando que es el aspecto que más se trabaja. Si bien el aspecto menos valorado es la formación recibida en ABP $(M=2.64)$, aspecto este, que es objetivo principal de esta investigación y la base para proponer futuras iniciativas de formación para el profesorado de Educación Infantil.

\subsubsection{Contraste de variables}

Al contar con una muestra con distintas características, podemos contrastar datos en función de ciertas variables categóricas, como por ejemplo, género, edad, años de experiencia laboral, titulación, titularidad del centro, curso en el que se imparte docencia, años que lleva trabajando con ABP. En este estudio la hipótesis nula $(H O)$ hace referencia a la no existencia de diferencias significativas asumiendo un valor de probabilidad de error de $\alpha=.050$ inferior, y la alternativa $(H 1)$ a la existencia de tales diferencias en las mismas condiciones.

Al contrastar la variable género con las dimensiones valoradas no se han encontrado diferencias estadísticamente significativas en ninguno de los aspectos consultados. Si bien, hemos podido comprobar que los docentes especialistas en Educación Infantil otorgan una mayor puntuación en todas dimensiones en contraposición con las valoraciones de los maestros de otra especialidad.

Otra variable que consideramos interesante contrastar es la edad de los docentes, dado que se suelen asociar las nuevas metodologías a profesorado de menor edad. El análisis exploratorio entre variables, utilizando 
el coeficiente de correlación de Pearson, da cuenta de una asociación entre la utilización del ABP y los años de experiencia profesional como docente. Los resultados muestra una relación positiva, moderada y estadísticamente significativa ( $r h o=0.43, p=0.000$ ). Las comparaciones post hoc, realizadas con el test de Scheffé, revelan que existen diferencias estadísticamente significativas entre los grupos de 26 a 35 años y 36 a 45 años con respecto a los docentes menores de 25 años y mayores de 55 años. De estos datos se desprende que los docentes muy jóvenes o muy mayores manifiestan reticencias a las nuevas metodologías.

Nos ha interesado contrastar los años de experiencia profesional y los años de trabajo con $A B P$, los datos de la Tabla 9 muestran que existen relaciones estadísticamente significativas entre la edad y las dimensiones objetivos-contenidos, metodología, evaluación, formación y TIC. En el caso de la relación de los años de trabajo con ABP y las dimensiones, obtenemos diferencias estadísticamente significativas en todos los casos excepto en formacióne innovación. Estos resultados revelan que cuantos más años se trabaja con ABP menos valoran la formación y la innovación en Educación Infantil.

\section{TABLA 9}

Resultados $r$ de Pearson entre las dimensiones valoradas y edad, años de experiencia y años de trabajo con ABP

\begin{tabular}{|c|c|c|c|c|c|c|c|c|c|}
\hline & \multirow[b]{2}{*}{$\begin{array}{c}\text { Años } \\
\text { expe- } \\
\text { riencia }\end{array}$} & \multirow[b]{2}{*}{$\begin{array}{c}\text { Años de } \\
\text { trabajo } \\
\text { con } \\
\text { ABP }\end{array}$} & \multicolumn{7}{|c|}{ ABP } \\
\hline & & & $\begin{array}{c}\text { Dise- } \\
\text { ño }\end{array}$ & $\begin{array}{l}\text { Obje- } \\
\text { tivos } \\
\text { conteni- } \\
\text { dos } \\
\end{array}$ & $\begin{array}{l}\text { Metodo- } \\
\text { logía }\end{array}$ & $\begin{array}{l}\text { Evalua- } \\
\text { ción }\end{array}$ & $\begin{array}{l}\text { Forma- } \\
\text { ción }\end{array}$ & TIC & $\begin{array}{l}\text { Innova- } \\
\text { ción }\end{array}$ \\
\hline \multirow{2}{*}{$\begin{array}{l}\text { Años experien- } \\
\text { cia } \\
\text { Años de traba- } \\
\text { jo con ABP } \\
\end{array}$} & 1 &, $255^{* *}$ &,- 134 &,- 047 &,- 032 &,- 031 & ,028 & ,016 & ,073 \\
\hline & & 1 &,- 009 & ,000 & ,022 &,- 043 & ,067 & ,043 & ,072 \\
\hline Diseño & & & 1 &, $173^{*}$ &, $252^{* *}$ &, $302 * *$ &, $223 * *$ & , 120 &,- 078 \\
\hline \begin{tabular}{|l|} 
Objetivos \\
contenidos
\end{tabular} & & & & 1 &, $360 * *$ &, $305^{* *}$ &, $345^{* *}$ &, 048 &,- 075 \\
\hline $\begin{array}{l}\text { Metodolo- } \\
\text { gía }\end{array}$ & & & & & 1 &, $582^{* *}$ &, $163^{*}$ & ,004 &,- 019 \\
\hline 率 Evaluación & & & & & & 1 &, $260 * *$ & ,047 &,- 090 \\
\hline Formación & & & & & & & 1 &, $384^{* *}$ &,- 107 \\
\hline TIC & & & & & & & & 1 &,- 078 \\
\hline Innovación & & & & & & & & & 1 \\
\hline
\end{tabular}

Hemos realizado la prueba $t$ de Student para determinar la influencia de la titularidad del centro porque la muestra participante solo ha sido de centros públicos y centros privados-concertados. Se puede observar en la Tabla 10, que existen diferencias estadísticamente significativas en 
valoraciones de las dimensiones formación y tecnología. Estos datos nos indican que los docentes de los centros públicos valoran más las dimensiones de formación y tecnología para el desarrollo del ABP que los de los centros privados-concertados.

TABLA 10

Resultados $t$ de Student entre las dimensiones valoradas y la titularidad del centro

\begin{tabular}{|l|c|c|}
\hline & $T$ & Sig. \\
\hline Diseño & -0.177 & 0.674 \\
\hline Objetivos y contenidos & -0.317 & 0.572 \\
\hline Metodología & -0.227 & 0.681 \\
\hline Evaluación & -1.868 & 0.175 \\
\hline Formación & -5.087 & 0.002 \\
\hline Tecnología & -8.750 & 0.003 \\
\hline Innovación & -1.019 & 0.891 \\
\hline
\end{tabular}

También hemos tenido en cuenta la formación específica en TIC que han recibido los participantes. Los datos de la Tabla 11, muestran diferencias estadísticamente significativas entre las valoraciones realizadas por los docentes en las dimensiones metodología, formación, tecnología e innovación. Hemos realizado las pruebas de Scheffé para detectar los grupos entre los que se dan diferencias y se observa en todos los casos que cuanto mayor es la formación en TIC más se valoran estas dimensiones.

\section{TABLA 11}

Resultados ANOVA de un Factor entre las dimensiones valoradas y el grado de formación en TIC.

\begin{tabular}{|l|c|c|}
\hline \multicolumn{1}{|c|}{} & $\mathrm{F}$ & Sig. \\
\hline Diseño & 1.906 & 0.125 \\
\hline Objetivos y contenidos & 0.856 & 0.513 \\
\hline Metodología & 4.231 & 0.001 \\
\hline Evaluación & 0.867 & 0.467 \\
\hline Formación & 2.563 & 0.007 \\
\hline Tecnología & 5.426 & 0.001 \\
\hline Innovación & 3.178 & 0.006 \\
\hline
\end{tabular}

Finalmente, dado que todos los docentes participantes utilizan ABP, nos pareció interesante contrastar la influencia del curso de Educación Infantil en el que imparte docencia actualmente. Los resultados de la Tabla 12 , indican que no existe relación significativa entre las dimensiones valoradas sobre el ABP y el curso en el que se imparte docencia. 


\section{TABLA 12}

Resultados ANOVA de un Factor entre las dimensiones valoradas y el curso en el que imparte docencia

\begin{tabular}{|l|c|c|}
\hline \multicolumn{1}{|c|}{} & $F$ & Sig. \\
\hline Diseño & 0.722 & 0.540 \\
\hline Objetivos y contenidos & 0.110 & 0.954 \\
\hline Metodología & 0.684 & 0.563 \\
\hline Evaluación & 1.056 & 0.369 \\
\hline Formación & 0.353 & 0.787 \\
\hline Tecnología & 0.610 & 0.609 \\
\hline Innovación & 0.984 & 0.405 \\
\hline
\end{tabular}

\section{DISCUSIÓN Y CONCLUSIONES}

En esta investigación se planteó como objetivo conseguir una fotografía de cómo el ABP está integrado en el proceso de enseñanza en la etapa de Educación Infantil. El desarrollo del mismo ha supuesto una situación ilustrativa de privilegio acerca de los beneficios y limitaciones que se derivan de la implementación del ABP.

Los resultados descritos anteriormente indican el nivel de conocimiento que dicen tener respecto al ABP los docentes que trabajan con este tipo de metodología, en concreto, en las dimensiones valoradas dicho conocimiento es "medio", a pesar de estar trabajando con este método. En cuanto a los aspectos de diseño de este tipo de metodología, manifiestan un conocimiento general, si bien se muestra más certero en la medida en que no se apoyan en editoriales para su diseño. También, consideran el ABP una herramienta innovadora, global e interdisciplinar, resultados coincidentes con los hallados en las investigaciones realizadas por Ortí e Iniesta-Sepúlveda (2016).

Asimismo, cabe destacar que según los propios docentes, el ABP se muestra como un instrumento muy efectivo para el desarrollo integral del alumnado en la medida que respeta tanto los ritmos de aprendizaje como sus intereses; como vemos, respetando además el origen social del aprendizaje (Vygotsky, 1982) y favoreciendo la justicia social (Bolívar, 2012; Latapí, 2012) durante el proceso de enseñanza. Para ello, es necesario abordar la totalidad de los elementos del ABP en la medida que permitan implementar esta metodología en coherencia con este nuevo paradigma pedagógico, donde la escuela se convierte en el contexto social en el que se desarrollan los procesos de enseñanza y aprendizaje. Por ello, se incide en la necesidad de establecer unos criterios claros para el diseño del ABP, una clara definición 
de los objetivos y contenidos, y de la evaluación a seguir. Además, cabe decir que de acuerdo con la literatura existente el ABP se plantea como una metodología pluridisciplinar que está directamente unida a los recursos tecnológicos y a la innovación.

Es necesario reconocer también, la dificultad que supone trabajar con ABP en términos de diseño, pues queda destacado el exiguo conocimiento de los docentes en términos de sus fundamentos psicológicos, pedagógicos y epistemológicos. En cuanto a la metodología, los datos resaltan la facilidad para modificar lo planificado, contrastando con la rigidez de la administración en este asunto. Y en lo que se refiere a la rigidez de la administración, vemos que trabajar con ABP ayuda a los docentes participantes a tratar contenidos "extra" a los exclusivamente curriculares.

Para finalizar, concluimos que asistimos a constantes cambios educativos que exigen una actualización permanente en todos los aspectos del desarrollo curricular. Por ello, desde la óptica metodológica, la capacidad de aceptar el cambio se concibe como un factor esencial para el óptimo desarrollo del sistema educativo, ya que se considera la herramienta que permite visualizar y evidenciar los avances en la materia; y esta adaptación, requiere flexibilización por parte de los docentes en aras de contribuir al desarrollo de nuevas competencias profesionales.

\section{REFERENCIAS}

Balongo González, E., y Mérida Serrano, R. (2016). El clima de aula en los proyectos de trabajo. Crear ambientes de aprendizaje para incluir la diversidad infantil. Perfiles Educativos, 38(152), 146-162.

Belavi, G., y Murillo, F. J. (2016). Educación, Democracia y Justicia Social. Revista Internacional de Educación para la Justicia Social (RIEJS), 5(1), 13-34 doi:10.15336/riejs2016.5.1

Bolivar, A. (2012). Justicia Social y equidad escolar. Una revisión actual. Revista Internacional de Educación para la Justicia Social, 1(1), 2012, pp. 9-45, ISSN: 2254-3139. doi: $10.15366 /$ riejs

Cascales-Martínez, A., Carrillo-García, M. E., y Redondo-Rocamora, A. M. (2017). ABPy Tecnología en Educación Infantil. Píxel-Bit. Revista de Medios y Educación, (50), 201-210. Recuperado de http://acdc.sav.us.es/ojs/index.php/pixelbit/article/view/903/815

Chávez Barquero, F. H., Cantú Valadez, M., y Rodríguez Pichardo, C. M. (2016). Competencias digitales y tratamiento de información desde la mirada infantil. Revista electrónica de investigación educativa, 18(1), 209-220. Recuperado de http://www.scielo.org.

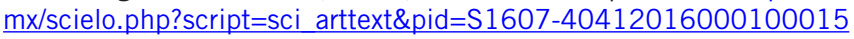

De Vellis, R.F. (2003). Scale development: Theory and applications ( $2^{\mathrm{a}}$ ed.). Thousand Oaks, CA: Sage 
Fernández Martínez, P., y Gallardo Fernández, I. M. (2016). “El lenguaje como medio de construcción social del conocimiento en educación infantil”. Revista Iberoamericana de Educación, 71, 111-131. Recuperado de: https://rieoei.org/RIE/article/view/7

Gallardo Fernández, I. M., Ríos García, I., Fernández Martínez, P., y Ramos Xavier, G. T. (2016). "Presentación. Educación Infantil (II)". Revista Iberoamericana de Educación, 72, 11-13. Recuperado de https://rieoei.org/RIE/article/view/43

Gay, L. R. \& P. Airasian (2000), Educational Research: Competencies for analysis and application, Upper Saddle River, NJ, Prentice-Hall.

Helm, J. H., \& Katz, L. G. (2016). Young investigators: The project approach in the early years. Teachers College Press. Recuperdado de https://books.google.es/books?hl=es\&lr $=\& i d=5 m Q L D A A A Q B A J \& o i=f n d \& p g=P P 1 \& d q=p r o j e c t+$ based + approach+in+ear $\mathrm{y}+\mathrm{childhood \& ots=kqbloLPRDt \& sig=PiHhaAXgd0bd5WAJC04fnDQ7w0w \# v=0ne}$ page\&q=project $\% 20$ based $\% 20$ approach\%20in $\% 20$ early\%20childhood\&f=false

Kilpatrick, W. H. (1929): The Project Method: The Use of the Purposeful Act in the Educative Process. Recuperado de https://archive.org/details/projectmethodusO0kilpgoog

Latapí Sarre, J. (2012). Educación y Justicia Social. Revista Internacional de Educación para la Justicia Social 1(1), 2012, pp. 199-202, ISSN: 2254-3139 Recuperado de http:// www.rinace.net/riejs/numeros/vol1-num1/doc1.pdf

Moral Pérez, M. E., Villalustre Martínez, L. y Neira Piñero, M. R. (2013). Oportunidades de las TIC para la innovación educativa en las escuelas rurales de Asturias. Aula Abierta, Vol. 42. Número 1. Enero-Junio 2014, 61-67.doi: 10.1016/S0210-2773(14)70010-1

Mosconi, E., Gamino, A., y González, A. (2016). El aula extendida, un espacio complementario integrador de conocimientos. Recuperado de http://ridaa.unicen.edu.ar/xmlui/ handle/123456789/509.

Ortí Martínez, J. A., y Iniesta-Sepúlveda, M. (2016). Didáctica de la Matemática a través de la Música y el Aprendizaje por Proyectos en Educación Infantil. Revista Internacional de Educación Preescolar e Infantil, 2(1), 31-40. Recuperado de http://journals. epistemopolis.org/index.php/eduinfantil/article/view/561

Ruiz Torres, M. D. C., y Mérida Serrano, R. (2016). Promover la inclusión de las familias a través del desarrollo de Proyectos de Trabajo. Un estudio de caso/Promoting the inclusion of families through the development of Work Projects.A case study. Revista Complutense de Educación, 27(3), 943. Doi: http://dx.doi.org/10.5209/ rev RCED.2016.v27.n3.47022

Vergara Ramírez, J. J. (2015). Aprendo porque quiero. El Aprendizaje Basado en Proyectos $(A B P)$, paso a paso. Editorial SM: Biblioteca Innovación Educativa.

Vygotsky, L. S. (1982). Obras escogidas. Tomo I, ed. Pedagóguika: Moscú; 1983. Traducción español. José María Bravo.

Vygotsky, L. S. (1982). Obras escogidas. Tomo II, ed. Pedagóguika: Moscú; 1983. Traducción español. José María Bravo.

Wieserma, L.D. (2001). Conceptualization and development of the sources of enjoyment in youth sport questionnaire. Measurement in Physical Education and Exercise Science, 5 (3), 153-157. 